\title{
Preliminary reference values for electrocardiography, echocardiography and myocardial morphometry in the European brown hare (Lepus europaeus)
}

\author{
Agnieszka Noszczyk-Nowak*1, Józef Nicpońn ${ }^{1}$, Marcin Nowak ${ }^{2}$ and \\ Piotr Slawuta ${ }^{1}$
}

Address: ${ }^{1}$ Department of Internal and Parasitic Diseases Veterinary Medicine Faculty, Wroclaw University of Environmental and Life Sciences, Wrocław 50-366, Poland and 2Department of Pathological Anatomy, Veterinary Medicine Faculty, Wroclaw University of Environmental and Life Sciences, Wrocław 50-366, Poland

Email: Agnieszka Noszczyk-Nowak* - agnieszkann@poczta.onet.pl; Józef Nicpoń - jozef.nicpon@up.wroc.pl; Marcin Nowak - marcin.nowak@up.wroc.pl; Piotr Slawuta - piotr.slawuta@up.wroc.pl

* Corresponding author

Published: 30 January 2009

Acta Veterinaria Scandinavica 2009, $51: 6$ doi:10.1 I86/I75I-0I47-5I-6

This article is available from: http://www.actavetscand.com/content/5I/l/6

(c) 2009 Noszczyk-Nowak et al; licensee BioMed Central Ltd.

This is an Open Access article distributed under the terms of the Creative Commons Attribution License (http://creativecommons.org/licenses/by/2.0), which permits unrestricted use, distribution, and reproduction in any medium, provided the original work is properly cited.
Received: 2 July 2008

Accepted: 30 January 2009

\begin{abstract}
The study aimed at defining reference values for electrocardiographic (ECG) and echocardiographic parameters as well as macroscopic dimensions of the heart and microscopic dimensions of cardiomyocytes in the European brown hare. The studies were conducted on 30 adult, clinically healthy hares of either sex caught in Poland. ECG and echocardiography were performed supravitally on anaesthetized hares. After euthanasia, gross and microscopic myocardial and cardiomyocyte dimensions were determined. Heart rate amounted to $140 \pm 37.5$ beats $/ \mathrm{min}$, the leading rhythm involved the sinus rhythm. $\mathrm{P}$ wave time was $26 \pm 5 \mathrm{~ms}$, PQ time was $80 \mathrm{~ms}$, QRS time was $29 \pm 3.5 \mathrm{~ms}$, and ST was $97.5 \pm 7 \mathrm{~ms}$. Echocardiography determined a left ventricular wall end-diastolic diameter of $8.6 \pm 2.0 \mathrm{~mm}$ and an intraventricular septum end-diastolic diameter of $5.75 \pm 1.0 \mathrm{~mm}$. The thickness of the interventricular septum corresponded to that of the free wall of the left ventricle, a finding consistent with physiological hypertrophy. Preliminary reference values were established for echocardiography. The findings were similar to those obtained at necropsy. The ECG and echocardiographic studies represent the first supravital examination of cardiac function in the hare. The obtained results illustrate adaptation of hare's myocardium to its mode of life. The cardiac findings resemble the athlete's heart syndrome described in humans. The findings may prove useful in further studies on the physiology of the cardio-vascular system in the hare.
\end{abstract}

\section{Findings}

Studies on the physiology of the European brown hare (Lepus europaeus) have focused on organ morphology, blood biochemical parameters, methods of blood sampling, coagulation parameters and cardiovascular disor- ders [1-6]. Physiological and morphological studies of the heart have not been performed, so the electrocardiographic (ECG) and echocardiographic variables remain unknown. Also, cardiomyocyte morphology remains to be reported. 
This study aims at defining reference values related to ECG and echocardiography and to determine gross and microscopic dimensions of the heart in the European brown hare.

The studies were conducted on 30 adult (body weight (BW) $3.2 \pm 0.54 \mathrm{~kg}$ ), clinically healthy hares (10 males and 20 females) out of 96 hares caught in south-eastern Poland. The 30 hares were selected randomly among the 96 hares by selecting every third clinically normal hare. Two hares were omitted from the sampling population due to low age and low BW, respectively. Examination of cardiac morphometry was done in 42 hares, including the 30 hares mentioned above, 8 hares euthanatized due to injuries to extremities, which had developed during transport and 4 hares, which died during the transport. The hares were euthanatized by phenobarbitaladministered intracardially.

The studies obtained consent of the $2^{\text {nd }}$ Local Ethical Commission, No.87/2006 (December 11, 2006). ECG and echocardiography were conducted following anesthesia by a mixture of xylazine (Sedazin, Biowet, Puławy, Poland) $3 \mathrm{mg} / \mathrm{kg}$ BW and ketamine (Bioketan, Vetoquinol Biovet, Gorzów Wielkopolski, Poland) $10 \mathrm{mg} / \mathrm{kg} \mathrm{BW,}$ administered intramuscularly.

ECG was conducted on animals positioned on their right flank, using a three-channel Sheiler AT-1 apparatus at the pass of $50 \mathrm{~mm} / \mathrm{s}$. On extremities the electrodes were placed in line with the generally accepted standards for small animals (Fig. 1) [7]. Amplitudes and duration of $\mathrm{P}$, $\mathrm{Q}, \mathrm{R}, \mathrm{S}, \mathrm{T}$ waves, QRS complex, time distances of PQ, QT, ST were measured in the second lead. Duration of $P$ wave

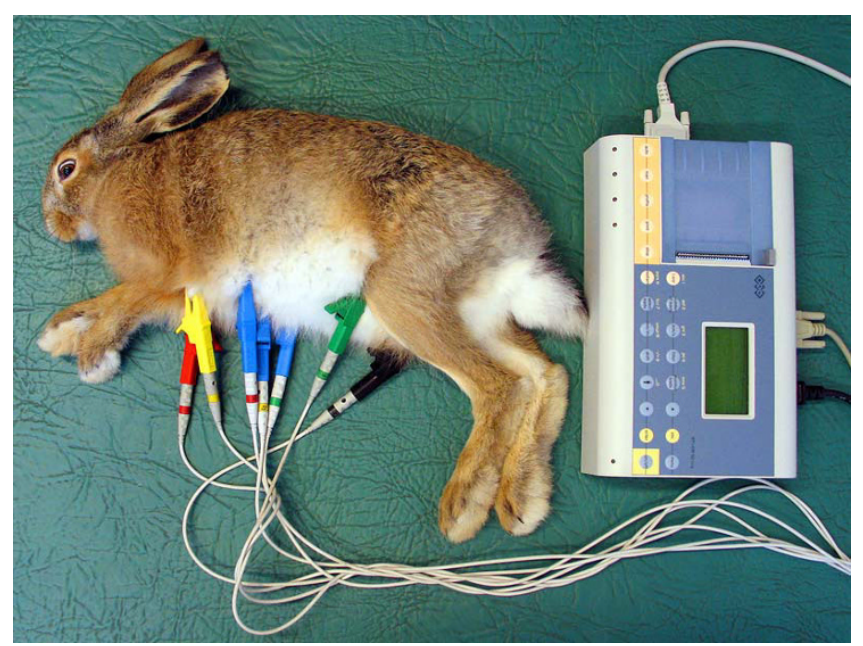

Figure I

Sites of electrode placement for electrocardiography in a European brown hare. was measured from the beginning of the rise to the end of the decrease in the record line. PQ (PR) interval was measured from the beginning of $\mathrm{P}$ wave to the beginning of QRS complex. QRS complex was measured from the beginning of $\mathrm{Q}$ wave to the end of $\mathrm{S}$ wave. QT interval was measured from the beginning of $\mathrm{Q}$ wave to the end of $\mathrm{T}$ wave. Q wave represents the first negative wave of QRS complex and in several species it is absent from ECG records. $\mathrm{R}$ wave represents the first positive wave of QRS complex, the descending arm of which below isoelectric line passes into the negative $S$ wave (Fig. 2). The mean electrical axis (MEA) was calculated on the basis of algebraic sum of QRS complex amplitudes in leads I and III plotted on the coordinate system. The mean electrical axis represents a direction of the resultant electromotive force of the heart and can be applied for diagnosing myocardial hypertrophy or disturbed intraventricular conductance. For every measured ECG parameter its mean value and standard deviation (SD) were calculated and the values allowed for calculation of relevant reference norms (mean \pm 2 SD) from 25 cycles. Data from all acral leads (I, II, III, aVR, aVL, aVF) were analyzed to detect disturbances in cardiac rhythm (Fig. 3).

The echocardiographic examination was performed using an Aloka 8000 apparatus equipped with a 7.5-10 Mzh head. Left ventricular end-systolic diameter, left ventricular end-diastolic diameter (LVEDd), left ventricular wall end-diastolic diameter (LWDd) and left ventricular wall end-systolic diameter in diastole as well as intraventricular septum end-diastolic diameter (IVSDd) and intraventricular septum end-systolic diameter were measured. The measurements were taken in parasternal projection in the short axis, from the right hand side, and the probe was

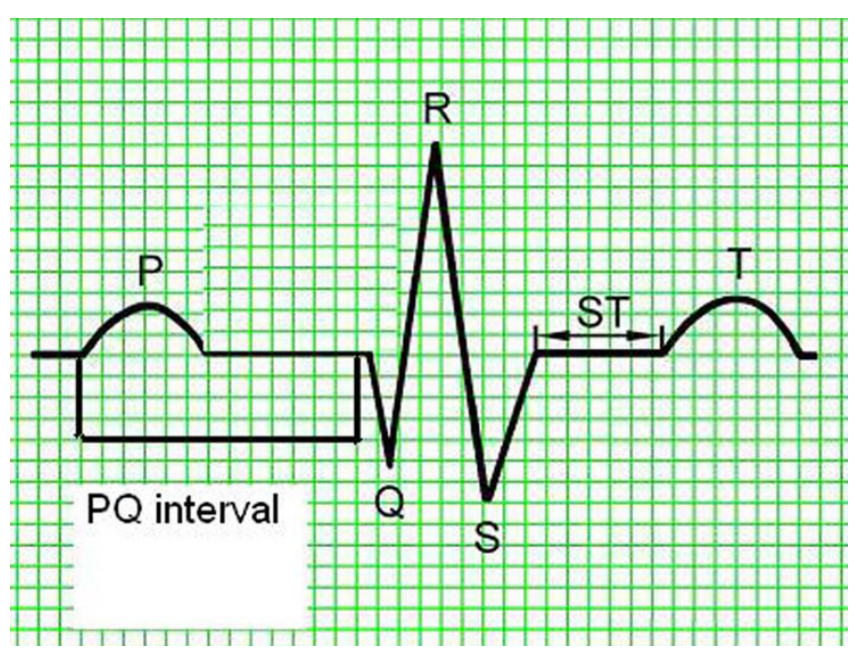

Figure 2

Schematic presentation of measured electrocardiography parameters. 


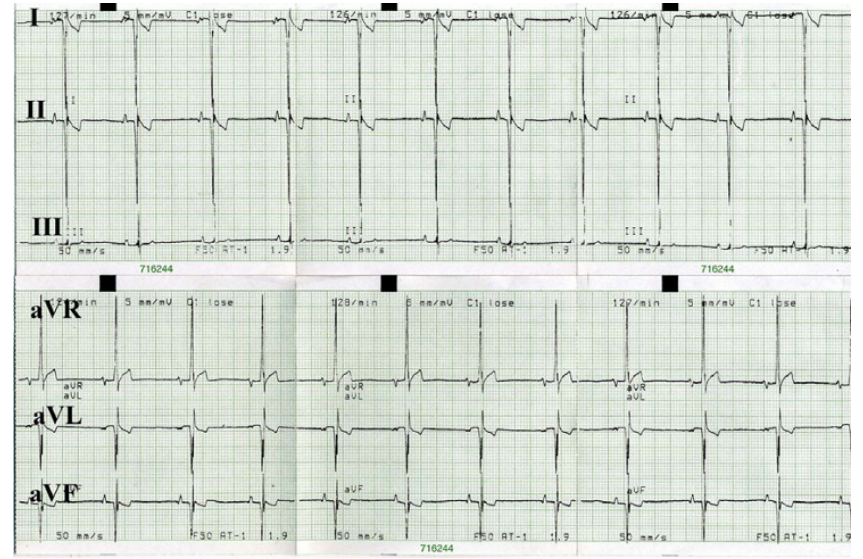

Figure 3

Electrocardiograph. Example of an electrocardiography recording in a European brown hare anaesthetized by xylazine and ketamine. Leads I, II, III, aVR, aVL, aVF are shown.

placed in the third and fourth intercostal space above the sternum [8]. The measurements allowed for automatic calculation of left ventricle ejection fraction and shortening fraction. Widths of aorta and of left atrium in vascular projection were estimated. For the obtained results, means and standard deviations were calculated and the data provided basis for calculation of reference values (mean \pm 2 SD). The relative wall thickness (RWT) was calculated as RWT $=$ IVSDd+LWDd/LVEDd. Examples of echocardiographic images are shown in Figures 4 and 5.

Post mortem examination of the cardiac morphometry included measurements of height and width of right and

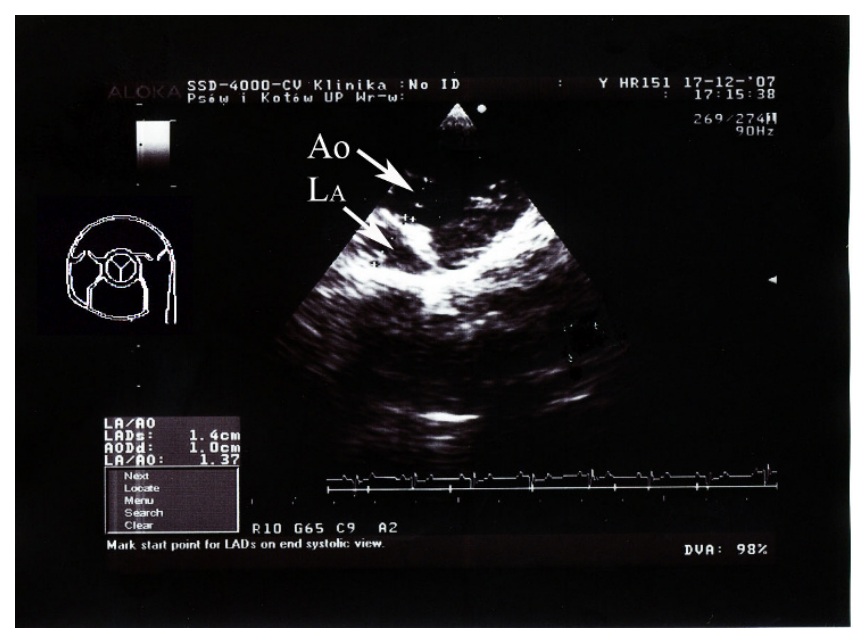

Figure 4

Echocardiographic image. Example of an echocardiographic examination (Vascular projection) in a European brown hare anaesthetized by xylazine and ketamine. Aorta: Ao, Left atrium (La).

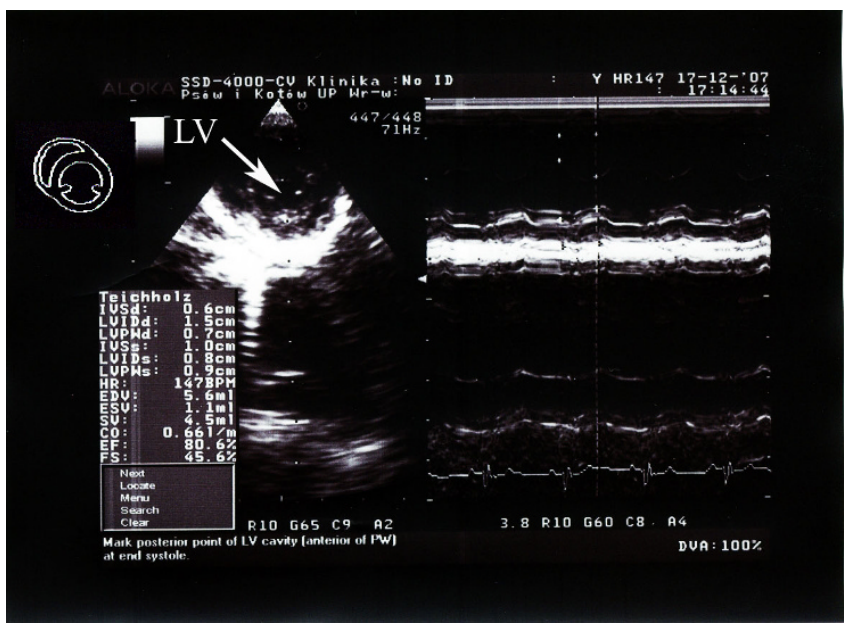

Figure 5

Echocardiographic image. Example of an echocardiographic examination in (Parasternal projection in the short axis) a European brown hare anaesthetized by xylazine and ketamine. Left ventricle: LV.

Table I: Obtained values (mean and standard deviation (SD)) and the corresponding determined reference values (mean $\pm 2 \times$ SD) for electrocardiographic parameters in European brown hares $(\mathbf{N}=30)$ anesthetized with xylazine and ketamine in the parasternal projection in short axis.

\begin{tabular}{lll}
\hline Parameter & Reference value & Mean and SD \\
\hline Heart rate (beats/min) & $100-178$ & $140 \pm 37.5$ \\
\hline P wave time (ms) & $16-36$ & $26 \pm 5$ \\
\hline P-wave amplitude (mV) & $0.14-0.42$ & $0.275 \pm 0.07$ \\
\hline PQ interval time (ms) & 80 & 80 \\
\hline QRS complex time (ms) & $22-36$ & $29 \pm 3.5$ \\
\hline Q-wave amplitude (mV) & UP to (-)3.2 & $(-) 2.4 \pm 0.4$ \\
\hline R-wave amplitude (mV) & Up to 5 & $1.925 \pm 1.55$ \\
\hline S-wave amplitude (mV) & Up to (-)0.2 & $0.1 \pm 0.05$ \\
\hline QT interval time (ms) & $100-160$ & $126 \pm 10.5$ \\
\hline ST interval time (ms) & $80-120$ & $97.5 \pm 7$ \\
\hline T-wave amplitude (mV) & Up to (-) 1.4 & $(-) 0.6 \pm 0.4$ \\
\hline Mean electrical axis (0) & $15-210$ & $97.5 \pm 113$ \\
\hline & & \\
\hline
\end{tabular}


left atrium, right and left ventricle, thickness of the interventricular septum and of the free wall in the left and right ventricle below the atrio-ventricular valves (similar to the site of measurement in echocardiographic examinations), and diameters of atrioventricular and arterial ostia. The locations are illustrated in additional file 1.

Tissue specimens of left ventricular free wall myocardium were fixed for $24 \mathrm{~h}$ in buffered $7 \%$ formalin, prepared by routine methods for histology, embedded in paraffin and sectioned at $4 \mu \mathrm{m}$. Sections were stained by hematoxylin and eosin and subjected to computer-assisted image analysis and morphometric measurements in a setup consisting of a computer connected to an Axiophot optical microscope (Carl Zeiss) equipped with a camera (model CC20P - Videotronic International). The entire set had the potential of recording images and of their digital analysis. The measurements took advantage of MultiScaneBase V $14.02 \mathrm{p}$ software, working in Windows environment. In every of 4 sections 10 optical fields (115 $\times 150 \mu \mathrm{m}$ ) were examined at $40 \times$ magnification. The morphometric analysis included measurements based on transverse as well as cross sections and included number of fibers per optical fields, diameter of cardiomyocytes and length and diameter of cardiomyocyte nuclei (see additional file 2) The obtained mean values with SD and the corresponding calculated reference values of ECG and echocardiographic parameters are presented in Tables 1 and 2, respectively. Gross and microscopic dimensions of the heart are shown in Table 3. Similar gross and microscopic dimensions were obtained independently of the cause of death (spontaneous death versus euthanasia).

The performed ECG and echocardiographic studies are the first supravital examinations of cardiac function in the hare. Even when anesthetic drugs were administered no disturbances were observed in cardiac rhythm or cardiac contractility. Anesthesia is needed to perform such studies in wild hares and xylazin-ketamine anesthesia provided a safe anesthesia [2].

The study demonstrated relatively thick ventricular walls and a relatively high ejection fraction thus reflecting the adaptation of hare's myocardium to their mode of life. The findings resemble the athlete's heart syndrome described in humans [9]. The pronounced and frequently repeated exertion leads to concentric hypertrophy of the myocardium without augmentation of cardiac cavities when the main inducing factor involves pressure load in the left ventricle [8]. Such cardiac transformation aims at securing increased stroke volume with preservation of the normal systolic function. The relative wall thickness in humans and in pigs amounts to $0.45[9,10]$. The relative wall thickness of $1.2 \pm 0.54$ found in this study points to cardiac hypertrophy in hares. No significant differences have been disclosed in thickness of interventricular septum and of free wall in the left ventricle, which indicates physiological hypertrophy [9]. The thickness of the inter-

Table 2: Obtained values (mean and standard deviation (SD)) and the corresponding determined reference values (mean $\pm 2 \times$ SD) for echocardiographic parameters in European brown hares $(\mathbf{N}=30)$ anesthetized with xylazine and ketamine in the parasternal projection in short axis.

\begin{tabular}{lll}
\hline Parameter $(\mathbf{m m})$ & Reference value & Mean value and SD \\
\hline Left ventricular end-systolic diameter & $6-20$ & $13.6 \pm 3.7$ \\
\hline Left ventricular end-diastolic diameter & $3.8-13.8$ & $8.8 \pm 2.5$ \\
\hline Left ventricular wall end-systolic diameter & $5.9-13.9$ & $9.9 \pm 2.0$ \\
\hline Left ventricular wall end-diastolic diameter & $6.6-10.6$ & $8.6 \pm 2.0$ \\
\hline Intraventricular septum end-systolic diameter & $2.4-12.4$ & $7.4 \pm 2.5$ \\
\hline Intraventricular septum end-diastolic diameter & $5.55-5.95$ & $5.75 \pm 1.0$ \\
\hline Left ventricular ejection fraction & $46.25-86.25$ & $66.27 \pm 9.9$ \\
\hline Shortening fraction & $18.95-48.75$ & $18.95-48.75$ \\
\hline Aorta & $4.35-11.15$ & $7.75 \pm 1.7$ \\
\hline Left atrium & $11.5-18.7$ & $15.12 \pm 1.8$ \\
\hline
\end{tabular}


Table 3: Gross and microscopic dimensions (mean and standard deviation (SD) of the heart of European brown hares ( $N=42$ ).

\begin{tabular}{|c|c|}
\hline Parameter & Mean value and SD \\
\hline Length of the heart $(\mathrm{mm})$ & $53.33 \pm 9.0$ \\
\hline Width of the heart $(\mathrm{mm})$ & $39.00 \pm 2.82$ \\
\hline Height of the right atrium $(\mathrm{mm})$ & $15.3 \pm 2.73$ \\
\hline Width of the right atrium $(\mathrm{mm})$ & $14.4 \pm 1.37$ \\
\hline Ring of tricuspid valve (mm) & $16.5 \pm 4.7 \times 13.16 \pm 4.62$ \\
\hline Height of the right ventricle $(\mathrm{mm})$ & $27.83 \pm 3.18$ \\
\hline Width of the right ventricle $(\mathrm{mm})$ & $27.66 \pm 4.84$ \\
\hline Myocardial thickness of the right ventricle free wall $(\mathrm{mm})$ & $4.66 \pm 0.5$ \\
\hline Pulmonary artery $(\mathrm{mm})$ & $6.16 \pm 1.1$ \\
\hline Height of the left atrium $(\mathrm{mm})$ & $11.16 \pm 7.5$ \\
\hline Width of the left atrium $(\mathrm{mm})$ & $12.3 \pm 3.0$ \\
\hline Ring of mitral valve $(\mathrm{mm})$ & $9.83 \pm 3.18 \times 11.05 \pm 1.55$ \\
\hline Height of the left ventricle (mm) & $31.83 \pm 4.91$ \\
\hline Width of the left ventricle $(\mathrm{mm})$ & $11.83 \pm 1.16$ \\
\hline Myocardial thickness of left ventricular free wall $(\mathrm{mm})$ & $8.66 \pm 1.5$ \\
\hline Myocardial thickness of the interventricular septum $(\mathrm{mm})$ & $8.16 \pm 1.3$ \\
\hline Aorta diameter $(\mathrm{mm})$ & $6.83 \pm 1.47$ \\
\hline Number of fibres in the assayed field amounted $(\mathrm{mm})$ & $40.52 \pm 7.26$ \\
\hline Diameter of cardiomyocytes in the ventricle $(\mu \mathrm{m})$ & $20.45 \pm 5.06$ \\
\hline Length of the cell nucleus $(\mu \mathrm{m})$ & $15.95 \pm 2.91$ \\
\hline Diameter of the cell nucleus $(\mu \mathrm{m})$ & $4.46 \pm 0.63$ \\
\hline
\end{tabular}

ventricular septum did not differ from that of the free wall in the left ventricle thus indicating physiological hypertrophy [9]. Present studies are, however, of a pioneer character and a larger group of the animals of various ages should be examined. The prominent ventricular myocardium is associated with high values of amplitudes in the QRS ventricular complex and of $Q$ wave in particular. Amplitude of $\mathrm{R}$ wave was also substantial, but with high SD. The study showed that hares have cardiomyocytes of a size similar to rabbits $[11,12]$.
Post mortem measurements and echocardiographic findings were similar thus demonstrating the usefulness of echocardiography to evaluate the heart of hares. Cardiac measurements were done on left and the right ventricular walls and of interventricular septum just below the atrioventricular valves.

The physiological studies based on ECG and echocardiography should be continued in order to verify the preliminarily established reference values. 


\section{Competing interests}

The authors declare that they have no competing interests.

\section{Authors' contributions}

ANN carried out of ECG and echocardiographic examinations, calculated the parameters, and drafted the manuscript. JN participated in the drafting and revised the content critically. $\mathrm{MN}$ performed the histopathological examinations. PS managed the anesthesia and necropsied the hares. All authors read and approved the final manuscript.

\section{Additional material}

\section{Additional file 1}

Illustrations showing the locations used to measure myocardial dimensions. a) transverse dimension, b) longitudinal dimension, c) right ventricle diameter, d) intraventricular septum diameter and e) left ventricle diameter.

Click here for file

[http://www.biomedcentral.com/content/supplementary/17510147-51-6-S1.jpeg]

\section{Additional file 2}

Micrographs showing the way cardiomyocytes were measured. a) crosssection and b) longitudinal section. Hematoxylin and eosin. Obj. $\times 40$ Click here for file

[http://www.biomedcentral.com/content/supplementary/17510147-51-6-S2.jpeg]

\section{References}

I. Marco I, Cuenca R, Pastor J, Velarde R, Lavin S: Hematology and serum chemistry values of the European brown hare. Vet Clin Pathol 2003, 32:195-198.

2. Nicpon J, Noszczyk-Nowak A, Slawuta P, Kozdrowski : Arterial and venous blood and urine collection techniques in European Brown Hares [in Polish]. Medycyna Wet 2008, 64:88-90.

3. Nicpon J, Sławuta P, Nicpon J, Noszczyk-Nowak A: Hematological, biochemical and acid-base equilibrium parameters of the European Brown Hare [in Polish]. Medycyna Wet 2007, 63:|239-124|.

4. Pikula J, Adam V, Bandouchova H, Beklova M, Horakova J, Horakova H, Kizek R, Krizkova S, Skocovska B, Supalkova V, Svoboda M, Tremi F, Vitula F: Blood coagulation times in the European brown hare (Lepus europaeus). Vet Clin Pathol 2007, 36:361-363.

5. Sargent AP: Spontaneous arteriosclerosis in a brown hare. J Wildl Dis 1974, 10:130-134.

6. Wright LJ: Spontaneous lesions in the aorta of the common brown hare (Lepus europaeus). Vet Pathol 1975, 1 2:37-45.

7. Szabuniewicz M, Hightower D, Kyzar JR: The electrocardiogram, vectocardiogram and spatiocardiogram in the rabbit. Can J Comp Med 1971, 35:107-I4.

8. Cheitlin M, Alpert J, Amstrong W: ACC/AHA guidelines for the clinical application of echocardiography. Circ 1997, 95:1686-1774.

9. Rich BS, Havens SA: The athletic heart syndrome. Curr Sports Med Rep 2004, 3:84-88.

10. Noszczyk-Nowak A, Pasławska U, Zysko D, Gajek J, Nicpoń J, Rabczyński J, Skrzypczak P: Cardiac hypertrophy induced by administration oral of L-thyroxine in growing pigs [in Polish]. Medycyna Wet 2007, 63:1 I3-II7.

II. Loughrey CM, Smith GL, MacEachern KE: Comparison of $\mathbf{C a}^{2+}$ release and uptake characteristics of the sarcoplasmic reticulum in isolated horse and rabbit cardiomyocytes. Am J Physiol Heart Circ Physiol 2004, 287: I 149-I I 59.
12. Zak R: Development and proliferative capacity of cardiac muscle cells. Circ Res 1974, 35(2):17-26.
Publish with Biomed Central and every scientist can read your work free of charge

"BioMed Central will be the most significant development for disseminating the results of biomedical research in our lifetime. "

Sir Paul Nurse, Cancer Research UK

Your research papers will be:

- available free of charge to the entire biomedical community

- peer reviewed and published immediately upon acceptance

- cited in PubMed and archived on PubMed Central

- yours - you keep the copyright
BioMedcentral 